\title{
Reduction of globotriaosylceramide in Fabry disease mice by substrate deprivation
}

\author{
Akira Abe, ${ }^{1}$ Susan Gregory, ${ }^{1}$ Lihsueh Lee, ${ }^{1}$ Paul D. Killen, ${ }^{2}$ Roscoe O. Brady, ${ }^{3}$ \\ Ashok Kulkarni, ${ }^{4}$ and James A. Shayman ${ }^{1}$ \\ ${ }^{1}$ Nephrology Division, Department of Internal Medicine, and \\ ${ }^{2}$ Department of Pathology, University of Michigan Medical Center, Ann Arbor, Michigan, USA \\ ${ }^{3}$ National Institute of Neurological Disorders and Stroke, and \\ ${ }^{4}$ National Institute of Dental and Craniofacial Research, National Institutes of Health, Bethesda, Maryland, USA \\ Address correspondence to: James A. Shayman, Nephrology Division, Department of Internal Medicine, \\ University of Michigan, Box 0676, Room 1560 MSRBII, 1150 West Medical Center Drive, Ann Arbor, \\ Michigan 48109-0676, USA. Phone: (734) 763-0992; Fax: (734) 763-0982; E-mail: jshayman@umich.edu.
}

Received for publication February 25, 2000, and accepted in revised form April 25, 2000.

We used a potent inhibitor of glucosylceramide synthase to test whether substrate deprivation could lower globotriaosylceramide levels in $\alpha$-galactosidase A ( $\alpha$-gal A) knockout mice, a model of Fabry disease. C57BL/6 mice treated twice daily for 3 days with D-threo-1-ethylendioxyphenyl-2palmitoylamino-3-pyrrolidino-propanol (D-t-EtDO-P4) showed a concentration-dependent decrement in glucosylceramide levels in kidney, liver, and spleen. A single intraperitoneal injection of D-t-EtDO-P4 resulted in a 55\% reduction in renal glucosylceramide, consistent with rapid renal glucosylceramide metabolism. A concentration-dependent decrement in renal and hepatic globotriaosylceramide levels was observed in $\alpha$-Gal $A^{-}$males treated for 4 weeks with D-t-EtDO-P4. When 8 -week-old $\alpha$-Gal $A^{-}$males were treated for 8 weeks with $10 \mathrm{mg} / \mathrm{kg}$ twice daily, renal globotriaosylceramide fell to below starting levels, consistent with an $\alpha$-galactosidase $\mathrm{A}$-independent salvage pathway for globotriaosylceramide degradation. Complications observed with another glucosylceramide synthase inhibitor, $N$-butyldeoxynojirimycin, including weight loss and acellularity of lymphatic organs, were not observed with D-t-EtDO-P4. These data suggest that Fabry disease may be amenable to substrate deprivation therapy.

J. Clin. Invest. 105:1563-1571 (2000).

\section{Introduction}

Fabry disease is a lysosomal storage disorder with clinical manifestations of renal failure, cardiovascular disease, neuropathy, and angiokeratomas. The disease is the result of the X-linked inherited loss of ceramide trihexosidase ( $\alpha$-glactosidase $A)$ activity leading to the accumulation of glycosphingolipids containing $\alpha$-galactosyl linkages in affected tissues (1). The primary glycosphingolipid detected is globotriaosylceramide (Gb3).

Gb3 contains glucosylceramide as its base cerebroside and in that regard is similar to the glycosphingolipids detected in several additional lysosomal storage disorders including Gaucher disease, Sandhoff disease, TaySachs disease, and GM1 gangliosidosis. Presently, only Gaucher disease has a proved and approved treatment. This therapy uses intravenous infusions of mannose terminated $\beta$-glucosidase (2). It is limited by its expense and lack of efficacy for forms of Gaucher with central nervous system involvement. A similar strategy has recently been applied in preliminary studies in Fabry disease using infused $\alpha$-galactosidase A (3).

An alternative treatment strategy, first proposed by Radin, is substrate deprivation (4). This approach is based not on the replacement of the defective glycosidase, but rather on the inhibition of an earlier step in the synthesis of the accumulating glycosphingolipid.
Promising results using this strategy have been reported using $N$-butyldeoxynojirimycin (NBDNJ), a compound originally developed as an $\alpha$-glucosidase inhibitor (5). NBDNJ was discovered to have moderate activity $\left(20 \mu \mathrm{M} \mathrm{IC}_{50}\right)$ as a glucosylceramide synthase inhibitor. In models of Tay-Sachs and Sandhoff disease, mice were shown to have delayed accumulation of ganglioside GM2, delayed onset of neurologic symptoms, and a modest prolongation of life $(6,7)$. NBDNJ, however, has limited activity as a glucosylceramide synthase inhibitor and thus must be given at high oral dosing $(0.6-4.8 \mathrm{~g} / \mathrm{kg} / \mathrm{d})$. As a result, NBDNJ-treated mice experience weight loss (8). In addition, there is a lymphopenic response to NBDNJ observed in both experimental animals and humans, manifest in part by a reversible decrease in spleen and thymus size. It is unclear whether the lymphopenia is the result of the $\alpha$ glucosidase or glucosylceramide synthase inhibition. D-Threo-1-Phenyl-2-decanoylamino-3-morpholinopropanol (PDMP) is a prototypic glucosylceramide synthase inhibitor (9). A series of more active homologues have been reported based on the diversification of its three functional groups including the fatty acid in amide linkage (10), the cyclic amine (11), and most recently the phenyl group (12). The phenyl group substitutions were designed based on Hansch analysis and 
resulted in the identification of two compounds that are 2,000-fold more active than is either NBDNJ or PDMP. These include the D-threo enantiomers of 1-ethylendioxyphenyl-2-palmitoylamino-3-pyrrolidinopropanol (D-t-EtDO-P4) and 1-4'-hydroxyphenyl-2palmitoylamino-3-pyrrolidino-propanol (D-t- $p \mathrm{OH}-\mathrm{P} 4)$. We recently reported that both PDMP homologues were capable of markedly depleting Gb3 in virally transformed lymphoblasts from a patient with Fabry disease (13). By contrast, NBDNJ displayed little if any activity. In the present study, we evaluated D-t-EtDO$\mathrm{P} 4$ in an in vivo model of Fabry disease, the $\alpha$-galactosidase A knockout mouse.

\section{Methods}

Glucosylceramide synthase inhibitors. D-Threo-1-ethylendioxyphenyl-2-palmitoylamino-3-pyrrolidinopropanol (EtDO-P4) and D-threo-1-4'-hydroxyphenyl2-palmitoylamino-3-pyrrolidino-propanol ( $p$-OH-P4) were synthesized by the Mannich reaction from 2-Nacylaminoacetophenone, paraformaldehyde, and pyrrolidine followed by reduction with sodium borohydride as detailed previously (12). Four enantiomers are produced during the synthesis. Because only the Dthreo enantiomers are active in inhibiting the glucosylceramide synthase, resolution of the active D-threo inhibitors is performed by chiral chromatography.

Mice/breeding. $\alpha$-Galactosidase A-deficient mice were generated by gene targeting a mutated allele and bred on a 129SVJ X C57BL/ 6 background (14). $\alpha-G a l A^{-}$males and $\alpha$-Gal $A^{-1-}$ females were provided by the National Institutes of Health, the genotypes confirmed, and a colony maintained at the University of Michigan. The mice were monogamously bred under standards established by the University Committee on the Use and Care of Animals.

Inhibitor administration. D-t-EtDO-P4 and D-t- $p \mathrm{OH}-\mathrm{P} 4$ are highly lipophilic compounds and thus require the use of a lipid vehicle or dispersant for injection. The use of corn oil, mouse albumin, and liposomes were studied as alternative vehicles. Unless otherwise stated, the glucosylceramide synthase inhibitor was used as a liposomal suspension using dioleoylphosphatidylcholine (DOPC; Avanti Polar Lipids, Alabaster, Alabama, USA). DOPC $(1.5 \mathrm{mg})$ was mixed with $0.2-2.0 \mathrm{mg}$ of $\mathrm{D}-t$ EtDO-P4 or D-t- $p$-OH-P4 in a $13 \times 100-\mathrm{mm}$ glass tube. The mixture was dried under a stream of nitrogen and dispersed into $2 \mathrm{~mL}$ of $0.9 \% \mathrm{NaCl}$ with a probe-type sonicator. The sonication was carried out for $14 \mathrm{~min}$ utes while the tube was placed in an ice water bath. The liposomes were prepared by repeated passage through a 23-gauge needle and filtration across a $0.2-\mu \mathrm{m}$ sterile filter (Acrodisc B; Gelman Sciences Inc., Ann Arbor, Michigan, USA). Recovery of the inhibitor after filtration was confirmed by ultraviolet spectrometry and exceeded 99\%. Two hundred microliters of liposomes were injected intraperitoneally with an insulin syringe.

Lipid extraction and analysis. Treated mice were sacrificed by cervical dislocation, and the organs were resected, weighed, and frozen in liquid nitrogen. The frozen tissues were stored at $-80^{\circ} \mathrm{C}$ before use. The frozen tissue $(0.05-0.25 \mathrm{~g})$ was homogenized in 0.75-0.95 $\mathrm{mL}$ of cold PBS with a glass homogenizer. The homogenate $(\sim 1 \mathrm{~mL})$ was transferred to a $16 \times$ 125-mm glass tube and mixed with $2.5 \mathrm{~mL}$ methanol and $1.25 \mathrm{~mL}$ chloroform. The mixture was sonicated for 10 minutes with a bath sonicator, maintained at room temperature for 1 hour, and centrifuged for 30 minutes at 2,000 $\mathrm{g}$. The supernatant was transferred into a second glass tube. The residue was suspended in $3.8 \mathrm{~mL}$ of chloroform/methanol/0.9\% $\mathrm{NaCl}$ (1:2:0.8), sonicated for 10 minutes, and kept at room temperature for 1 hour. The suspension was centrifuged as already described here. The supernatants were combined and mixed with chloroform $(2.25 \mathrm{~mL})$ and $0.9 \%$ $\mathrm{NaCl}(2.25 \mathrm{~mL}$, wt/vol) using a vortex mixer. After centrifugation at $800 \mathrm{~g}$ for 5 minutes, the upper layer was discarded and the lower layer was washed with $2.25 \mathrm{~mL}$ of methanol and $1.8 \mathrm{~mL}$ of $0.9 \% \mathrm{NaCl}$. The lower layer was washed again with $2.25 \mathrm{~mL}$ methanol and $1.8 \mathrm{~mL}$ of $0.3 \% \mathrm{NaCl}$. The resultant lower layer was transferred into another tube and dried under a stream of nitrogen gas. The dried lipid was dissolved in chloroform/methanol $(2: 1)$ and stored at $-20^{\circ} \mathrm{C}$ before analysis. The total phospholipid content was determined by the method of Ames (15).

For the analysis of neutral glycosphingolipids from liver, kidney, and heart, a volume of lipid extract corresponding to $1 \mu \mathrm{mol}$ of total phospholipid was placed into a $16 \times 125-\mathrm{mm}$ glass tube and dried under a stream of nitrogen gas. The dried lipid was redissolved in chloroform and subjected to alkaline methanolysis and acid hydrolysis as described previously (13). The hydrolyzed lipids were used for analysis of glucosylceramide (500-700 nmol total phospholipid phosphate for kidney and liver; 100 nmol for spleen) and Gb3 (50-200 nmol total phospholipid) by thin layer chromatography. Borate impregnated plates were used for glucosylceramide analysis. For both glucosylceramide and Gb3, two solvents were utilized. Plates were first developed in a solvent of chloroform/methanol (98:2) and air dried in a fume hood. The plates were then developed in chloroform/methanol/acetic acid/methanol (61:33:3:3) and chloroform/methanol/water (16:6:1) for separations of Gb3 and glucosylceramide, respectively. The plates were then air dried, sprayed with $8 \%$ (wt/vol) $\mathrm{CuSO}_{4}$ pentahydrate in water/methanol/conc. $\mathrm{H}_{3} \mathrm{PO}_{4}(60: 32: 8)$ and charred for 15 minutes at $150^{\circ} \mathrm{C}$. The plates were scanned with a Kodak digital camera (Eastman Kodak Co., Rochester, New York, USA) and analyzed with the NIH Image 1.49 program (National Institutes of Health, Bethesda, Maryland, USA) to measure the density of each band. Glycosphingolipids were quantified by comparison to known standards.

Due to the very low level of glucosylceramide compared to galactosylceramide in mouse brain, analysis required the prior separation with a silica gel column. The lipid extract corresponding to $2 \mu \mathrm{mol}$ of phospholipid phosphate was dried in a $16 \times 125-\mathrm{mm}$ glass 

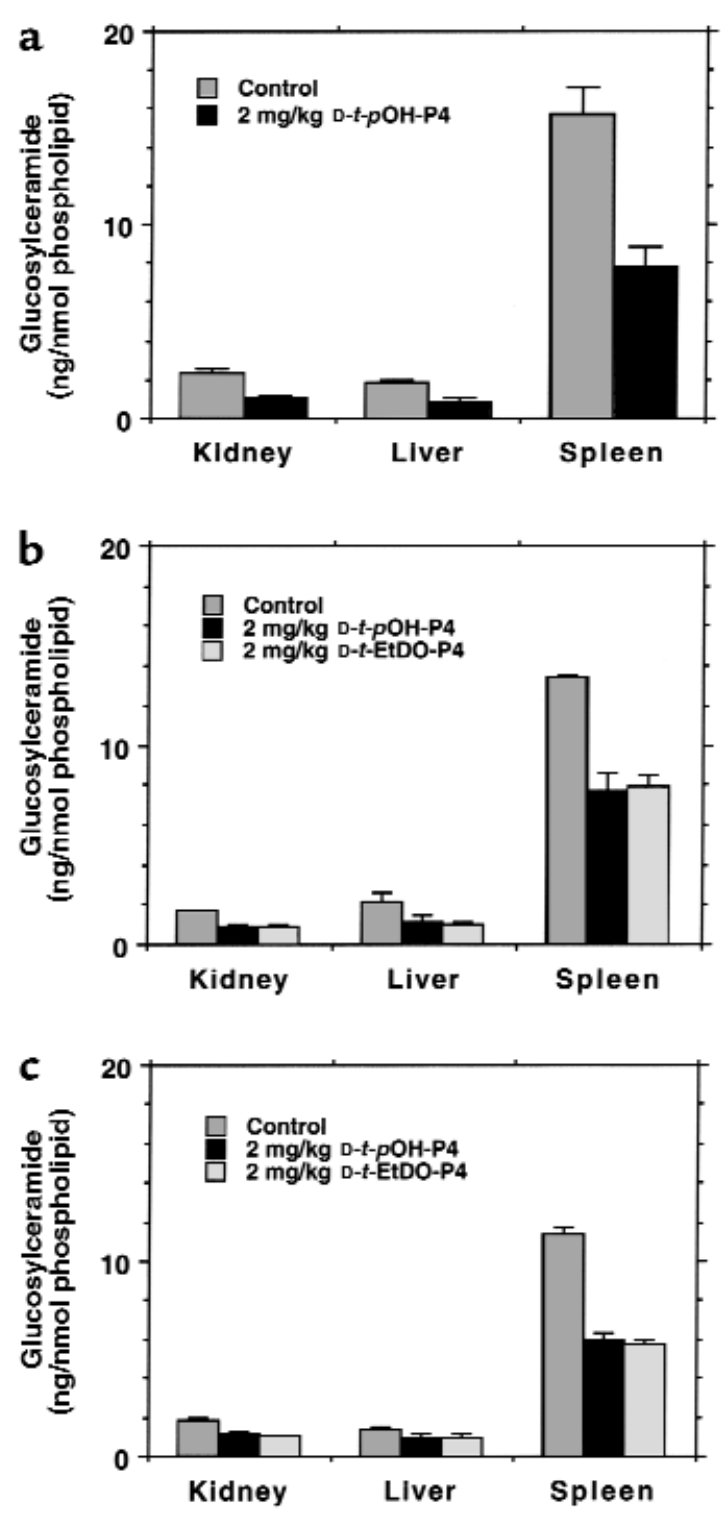

Figure 1

Organ levels of glucosylceramide using alternative vehicles for D-tEtDO-P4 and D-t-pOH-P4 dispersion in C57BL/ 6 mice. (a) Corn oil; (b) delipidated mouse albumin; (c) DOPC liposomes. The data represent the mean $\pm \mathrm{SD}$ ( $n=3$ for condition and determination). The differences for each inhibitor using each vehicle were significant at $P<0.01$ with the exception of liver glucosylceramide levels for albumin and DOPC liposomes. In these cases, $P<0.05$.

tube under nitrogen gas. The dried lipid was resuspended in chloroform and subjected to alkaline methanolysis as already described here. The hydrolyzed lipids were dissolved in $500 \mu \mathrm{L}$ of chloroform and applied to a silica gel column preequilibrated with chloroform (bed volume, $1 \mathrm{~mL}$ ). A stepwise elution consisting of chloroform $(5 \mathrm{~mL})$, chloroform/methanol (98:2; $5 \mathrm{ml})$, chloroform/methanol (95:5; $10 \mathrm{~mL}$ ), and chloroform/methanol (90:10; 10 $\mathrm{mL}$ ) was performed. Borate-impregnated plates were developed in a two-solvent system. The solvents were chloroform/methanol (98:2) and chloroform/methanol/water (60:30:6). The same charring and densitometry protocols already described here were used.

Flow cytometry. Splenocytes were isolated by placing freshly resected spleens in $3 \mathrm{~mL}$ DMEM and 5\% FBS. Cells were removed by pressing the spleens against the bottom of a Petri dish, and cellular clumps were dispersed by repeated passage through a 19-gauge needle. Cells were then filtered through a 200- $\mu \mathrm{m}$ mesh nylon screen and centrifuged for 10 minutes at $200 \mathrm{~g}$. This process was repeated once with media and twice more with lysis buffer. A total of $5 \times 10^{5}$ cells per sample were incubated on ice with FITC-conjugated antibodies (Sigma Chemical Co., St. Louis, Missouri, USA), including anti-mouse CD4 (H129.19), anti-mouse CD8a (53-6.7), and antimouse kappa light chain (EM 34.1). A FITC-conjugated IgG2a isotype was used as a control. Measurements were performed on a Beckman-Coulter Epics Elite ESP cell sorter (Beckman Coulter Inc., Miami, Florida, USA) through the flow cytometry core laboratory at the University of Michigan.

Blood urea nitrogen measurements were performed by the Unit for Laboratory Animal Medicine at the University of Michigan using a Kodak Ektachem DT60 Analyzer (Eastman Kodak Co.)

Electron microscopy. Fresh tissue was minced in 1-mm cubes and fixed by immersion in $4 \%$ glutaraldehyde, 0.1 $\mathrm{M}$ sodium cacodylate buffer ( $\mathrm{pH}$ 7.3). The tissue was post-fixed with osmium tetroxide before embedding in Epon. One-micron sections, stained with toluidine blue, were screened by light microscopy to select cross sections of tubules for ultrastructural study. Thin sections were stained with uranyl acetate and lead citrate before examination with a Philips 400T transmission electron microscope. The ultrastructure was blindly assessed by two independent observers and representative photomicrographs were selected.

\section{Results}

For all of the experiments the glucosylceramide synthase inhibitors were administered intraperitoneally. The P4 homologues are hydrophobic and therefore disperse poorly in aqueous solutions. Three alternative vehicles were sought for appropriate delivery of drug. These included dispersion in corn oil, delipidated mouse serum albumin, and DOPC liposomes. The glucosylceramide contents of kidney, liver, and spleen were measured in wild-type mice after administration of $2 \mathrm{mg} / \mathrm{kg}$ every 12 hours for 3 days. The mice were sacrificed 12 hours after the final dose. In each case, significant decrements in glucosylceramide were observed (Figure 1). The corn oil, however, was retained in the peritoneal cavities of the mice. In excess of $600 \mu \mathrm{L}$ remained after the final injection. In addition, the tissue glucosylceramide levels were higher for the control animals, raising the possibility that the corn oil itself increased the basal levels of the cerebroside. The mouse albumin was a highly effective 

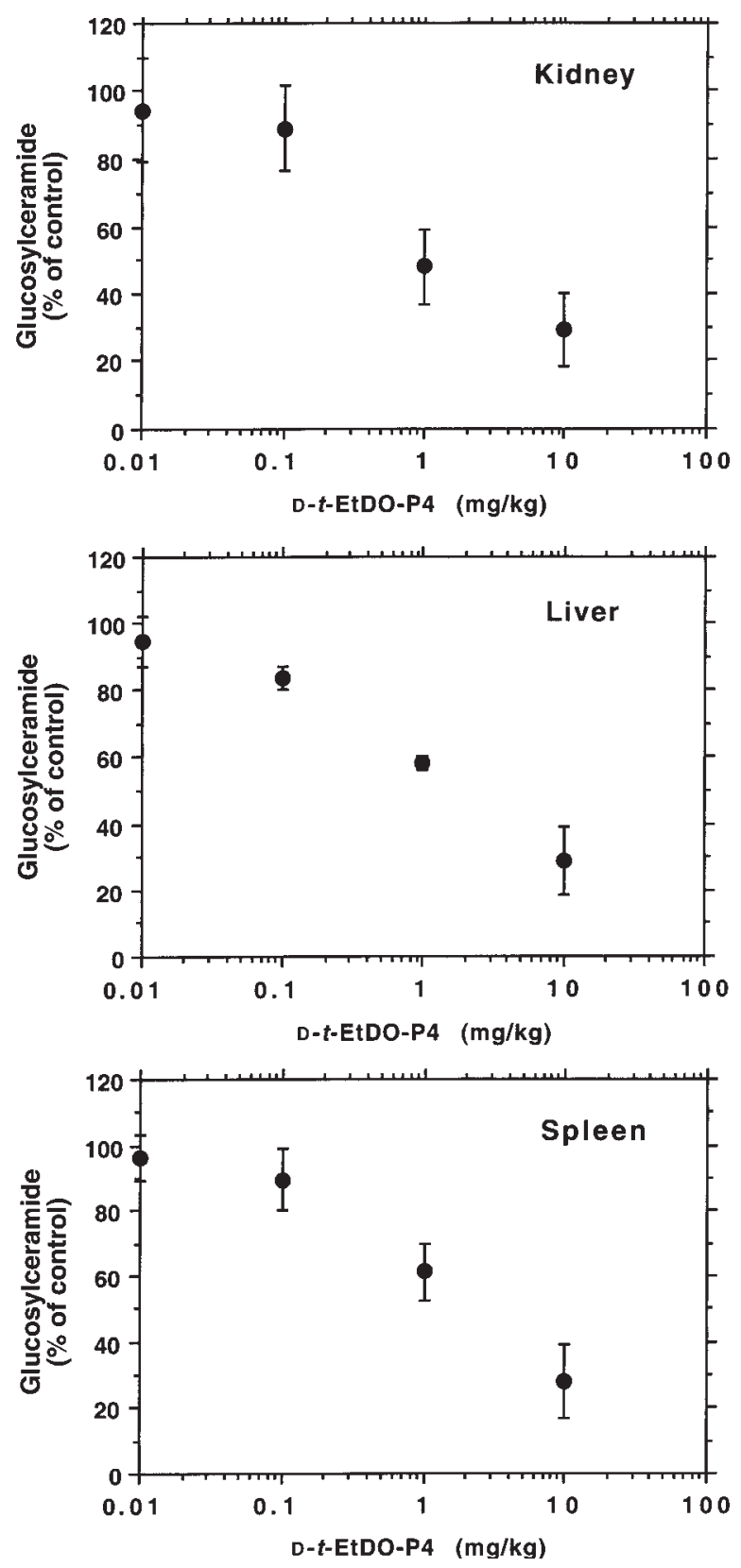

Figure 2

Concentration-dependent effects of D-t-EtDO-P4 on kidney, liver, and spleen glucosylceramide levels in C57BL/ 6 mice. The glucosylceramide synthase inhibitor was administered in a DOPC liposomal complex every 12 hours for 3 days. Mice were sacrificed 12 hours after the sixth injection ( $n=3$ for each time point).

vehicle but was not further used owing to its prohibitive cost. Therefore, DOPC liposomes were used for all further experiments.

The concentration-dependent effects of D-t-EtDO-P4 on kidney, liver, and spleen glucosylceramide content were evaluated (Figure 2). Wild-type mice were treated for 3 days with inhibitor every 12 hours intraperitoneally. The effect on kidney glucosylceramide was the most sensitive to D-t-EtDO-P4 with a $50 \%$ reduction at a dose of $1 \mathrm{mg} / \mathrm{kg}$. In all tissues a $75-80 \%$ reduction of glucosylceramide was observed with $10 \mathrm{mg} / \mathrm{kg}$.
The sensitivity of kidney to glucosylceramide synthase blockade was confirmed by evaluating the change in cerebroside levels in response to a single injection of D-t-EtDO-P4 (10 mg/kg) (Figure 3). Kidney and liver glucosylceramide levels were measured at $0,1,3,6,12$, and 24 hours after drug administration. A statistically significant decrement in renal glucosylceramide was observed as early as 6 hours after injection. A significant fall in hepatic glucosylceramide was not observed until 24 hours following treatment. These data were consistent with a very rapid turnover of renal glucosylceramide.

The natural history of Gb3 accumulation in the major affected organs of $\alpha$-Gal $A^{-}$males was determined (Figure 4). In contrast to C57BL/6 mice, in which only organ levels of renal Gb3 are detectable by the lipid extraction and charring methods used in this study (1.4 ng/nmol phospholipid), Gb3 was easily detectable in the kidneys, livers, and hearts of the homozygous females and hemizygous males. Gb3 levels were greater in kidney than in liver and heart, consistent with the original description of this model. The rate of accumulation was most significant between 4 and 16 weeks of age, with persistent accumulation still observed at 52 weeks of age.

The effect of a short-term, 3-day treatment with 2 $\mathrm{mg} / \mathrm{kg} \mathrm{D}-\mathrm{t}$-EtDO-P4 on the Gb3 and glucosylceramide levels in $\alpha$-Gal $A^{-}$males was assessed. Inhibitor treatment resulted in a significant loss of renal, liver, and splenic glucosylceramide but no detectable changes in tissue Gb3 levels was observed (Figure 5). A more prolonged treatment with D-t-EtDO-P4 was therefore conducted.

Eight-week-old $\alpha$-Gal $A^{-}$males were treated with 2, 10,15 , or $30 \mathrm{mg} / \mathrm{kg}$ of D-t-EtDO-P4 every 12 hours intraperitoneally for 4 weeks. Gb3 content of kidney and liver was significantly lower than liposome-treated control mice after the 4-week treatment (Figure 6a). Animals treated with either 2, 10, or $15 \mathrm{mg} / \mathrm{kg}$ of inhibitor demonstrated no adverse effects in feeding behavior or activity. No significant change in total body weight was observed for those mice treated with up to $15 \mathrm{mg} / \mathrm{kg}$ of inhibitor. However, mice treated with $30 \mathrm{mg} / \mathrm{kg}$ were observed to lose weight and were therefore sacrificed before the end of the 4-week treatment period. For both kidney and liver, a significant concentration-dependent decrement in Gb3 was observed (Figure 6b). Of significance was the observation that the renal Gb3 levels after 4 weeks of treatment with 10 or $15 \mathrm{mg} / \mathrm{kg}$ were significantly lower than those observed in the 8-week-old mice (treatments of $22.4 \pm 0.75$ vs. $18.2 \pm 2.47$ and $15.4 \pm$ $1.73 \mathrm{ng} / \mathrm{nmol}$ phospholipid for 10 and $15 \mathrm{mg} / \mathrm{kg}$ every 12 hours, respectively). This suggested that the inhibitor was lowering Gb3 to levels below those that the animals had accumulated.

To confirm this observation, 8-week-old $\alpha$-Gal $A^{-}$ males were treated for 8 weeks with D-t-EtDO-P4 10 $\mathrm{mg} / \mathrm{kg}$ every 12 hours. No change in body weights for D-t-EtDO-P4-treated versus control mice was 

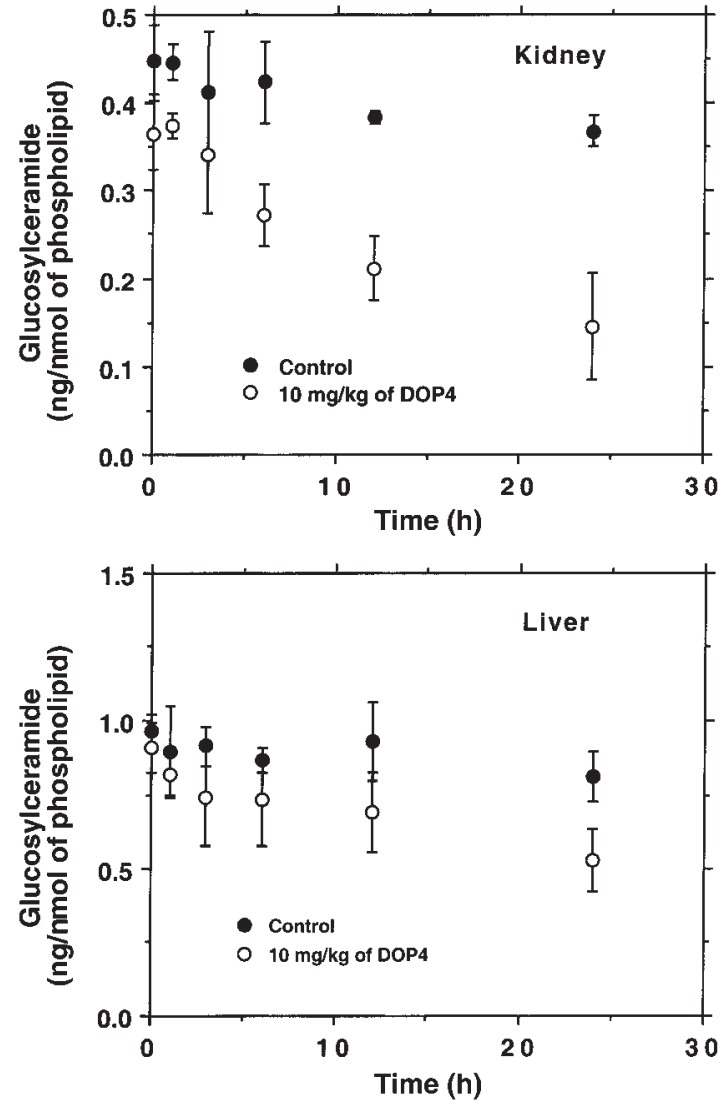

Figure 3

Time-dependent changes in glucosylceramide content of kidney and liver in C57BL/ 6 mice. A single intraperitoneal injection of DOPC liposomes or a D-t-EtDO-P4 (10 mg/ $/ \mathrm{kg})$ liposomal complex was administered intraperitoneally, and the mice were sacrificed at 1,3 , 6,12 , or 24 hours after injection ( $n=3$ for each point). For kidney measurements, $P<0.01$ at 6,12 , and 24 hours by the paired $t$ test. For liver measurements, $P=0.022$ at 24 hours.

observed when measured daily for 8 weeks (Figure 7a). Additionally, organ weights, including those of spleen and thymus, were not significantly lower in the inhibitor-treated animals compared with controls (Figure 7b). The blood urea nitrogen levels were compared between treated and control animals and were not significantly different $(22.0 \pm 1.9$ vs. $21.8 \pm 1.3$ $\mathrm{mg} / \mathrm{dL}$ ). Because another glucosylceramide synthase inhibitor, NBDNJ, had been previously reported to induce lymphopenia in association with a decrease in spleen and thymus weights, the distribution of lymphocytic markers was determined in splenocytes subjected to flow cytometry. The percent of cells expressing of CD4, CD8, or kappa light chain markers were $14.8,14.6$, and 35.7, respectively, in control splenocytes, and 15.1, 12.2, and 40.8, respectively, in mice treated with D-t-EtDO-P4 $10 \mathrm{mg} / \mathrm{kg}$ every 12 hours for 4 weeks. Thus, no changes in either the lymphatic organ cellularity or in the distribution of CD4, CD8, or kappa light chain expression were detected.

Gb3 levels in kidney, liver, and heart were significantly depressed with inhibitor treatment at both 4 and 8 weeks of treatment (Table 1). Renal Gb3 levels at 8 weeks of treatment were lower than those observed for 4 weeks of treatment and significantly below those observed for 8 -week-old mice at the onset of treatment ( $22.4 \pm 0.75$ vs. $15.3 \pm 1.73 \mathrm{ng} / \mathrm{nmol}$ phospholipid) (Figure 8).

Gb3 does not accumulate in the brains of $\alpha$-Gal $A^{-}$ males. Therefore, brain glucosylceramide levels were also measured in 8-week-old $\alpha$-Gal $A^{-}$males treated with D-t-EtDO-P4 $10 \mathrm{mg} / \mathrm{kg}$ every 12 hours for 8 weeks. Brain glucosylceramide levels are one-third those found in kidney and one-sixth those measured in liver. Nevertheless, a small yet consistent decrease in glucosylceramide content was observed ( 0.175 vs. $0.147 \mathrm{ng}$ glucosylceramide/nmol phospholipid in control versus treated mice; $n=2$ ).

The ultrastructural changes in the kidneys of the untreated, liposome-treated, and D-t-EtDO-P4-treated mice were also evaluated. The examination of semithin sections $(1 \mu \mathrm{m})$ failed to reveal consistent differences between any of the specimens. In particular, no vacuolar changes were evident in glomerular podocytes or tubular cells. However, ultrastructural examination of the kidneys from saline- or liposome-treated mice revealed numerous lamellated lipid-rich inclusions in proximal and distal tubular epithelial cells and to a lesser extent in podocytes. These myeloid bodies were scattered throughout the cell cytoplasm but were prominently clustered in the apical portions of the proximal tubular epithelial cells (Figure 9a). In addition to the small clustered myeloid bodies, large lipidladen inclusions were evident within the cytoplasm of these cells (Figure 9b). By contrast, renal tissue from D$t$-EtDO-P4-treated mice were distinguished from the control samples by the virtual absence of these large inclusions and by the presence of clusters of small myeloid bodies restricted to the apical aspect of the proximal tubular epithelial cells (Figure 9c).

\section{Discussion}

Fabry disease is an X-linked deficiency of $\alpha$-glactosidase A for which there is currently no proved or approved treatment. Between 6,000 and 10,000 affected individuals are believed to suffer from Fabry disease,

Table 1

Organ Gb3 content after 4- or 8-week treatment with D-t-EtDO-P4

Treatment Globotriaosylceramide (ng/nmol phospholipid)

\section{DOPC liposomes}

4 weeks $(n=5)$ Kidney Liver Heart ${ }^{\mathrm{A}}$ 8 weeks $(n=7) \quad 29.6 \pm 1.95 \quad 16.3 \pm 1.52 \quad 6.21 \pm 0.03$

D-t-EtDO-P4

( $10 \mathrm{mg} / \mathrm{kg}$ every 12 hours)

$\begin{array}{llll}4 \text { weeks }(n=5) & 18.2 \pm 2.47^{\mathrm{B}} & 8.46 \pm 0.87^{\mathrm{B}} & \\ 8 \text { weeks }(n=7) & 15.3 \pm 1.73^{\mathrm{B}} & 7.90 \pm 0.47^{\mathrm{B}} & 3.78 \pm 0.24^{\mathrm{B}}\end{array}$

${ }_{n}=4$ for heart measurements. ${ }^{B} P<0.01$ DOPC liposomes versus D- $t$-EtDO-P4 


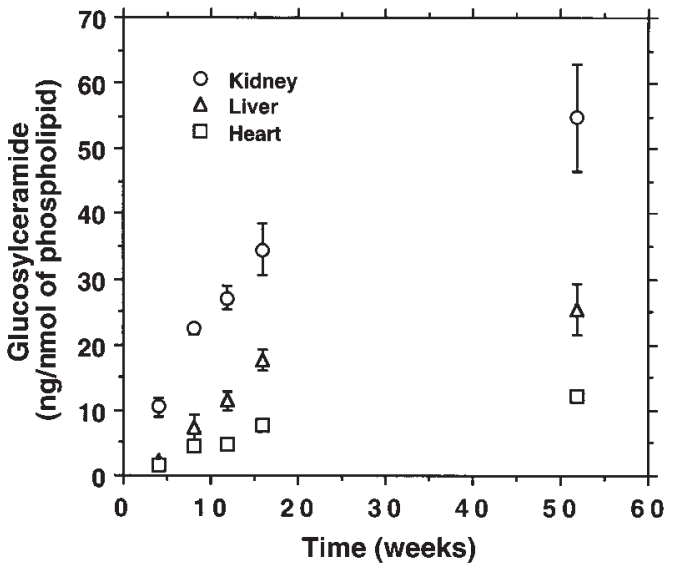

Figure 4

Age-dependent changes in Gb3 content of kidney, liver, and heart in $\alpha$-Gal $A^{-}$males. Data represent Gb3 determinations in three to five per time point and are expressed as the mean \pm SD.

with death occurring prematurely due to cardiovascular complications and renal failure. Several therapeutic approaches have been suggested including enzyme infusion, bone marrow transplantation, and gene therapy. Enzyme infusion of $\alpha$-galactosidase A was shown to decrease the liver and shed renal tubular cell Gb3 content in 10 patients with Fabry disease (3).

There exists limited experimental support for substrate depletion as a general strategy for treating glycosphingolipidoses. Liu et al. crossed Sandhoff mice, those lacking hexosaminidase $\mathrm{B}$, with those deficient in $\beta-1,4$-acetylgalactosaminyltransferase. The homozygous null offspring displayed a prolonged life span and a delay in the onset of neurologic symptoms. This form of experimental epistasis demonstrated that animals could survive in the absence of formation of de novo ganglioside GM2 synthesis and benefit from genetically engineered substrate deprivation (16).

NBDNJ has been reported to have beneficial effects in blocking the manifestations glycosphingolipid accumulation in murine models of Tay-Sachs and Sandhoff diseases $(6,7)$. Mice treated with oral dosing between 0.6 and 1.8 $\mathrm{gm} / \mathrm{kg} / \mathrm{d}$ demonstrated a $10-20 \%$ decline in body weight and a 50\% fall in spleen and thymus weights consistent with the development of acellular lymphoid tissues. Because these animals were not pair fed, the nutritional effects on the parameters studied, including longevity and glycosphingolipid metabolism, were indeterminate. The differences in caloric intake between the NBDNJ-treated and control animals may be important, as in other experimental models caloric restriction is associate with prolonged life span and significant impairment of sphingomyelin as well as neutral and acidic glycosphingolipid synthesis (17). In some cases, this is specifically attributable to deficiencies in thiamine or vitamins $A$ and $\mathrm{K}(18,19)$.

The prototypic glucosylceramide synthase inhibitor, PDMP, also displays significant toxicities. In vivo studies demonstrated that PDMP had significant short- term effects on renal growth, as well as short-term neurologic effects (20). In vitro studies demonstrated that PDMP significantly impaired cell growth in association with a rise in ceramide levels that was concurrent with the depletion of glucosylceramide (21). The cell growth inhibition was shown to be the result of cell-cycle blockade and could be replicated by the addition of cell-permeant ceramides to these culture systems.

The increase in cell ceramide levels was interpreted to be the result of substrate accumulation resulting from glucosylceramide synthase inhibition. However, with the synthesis and evaluation of various PDMP homologues, it was observed that the ceramide accumulation could be readily dissociated from the glucosylceramide depletion. For example, the threo and erythro diastereomers of the palmitoylamino- and pyrrolidino-substituted PDMP homologues were equally effective in raising ceramide levels and blocking cell proliferation, but only the threo diastereomer depleted glucosylceramide (11). A homologue in which an aliphatic group was substituted for the phenyl group displayed moderate glucosylceramide synthase inhibition in the absence of ceramide accumulation (22). One conclusion drawn from these structure activity studies was that PDMP was acting at a second site of ceramide metabolism. This alternative pathway may be the acylation of ceramide at the 1-hydroxyl $(23,24)$.

The recognition that PDMP homologues could dif-
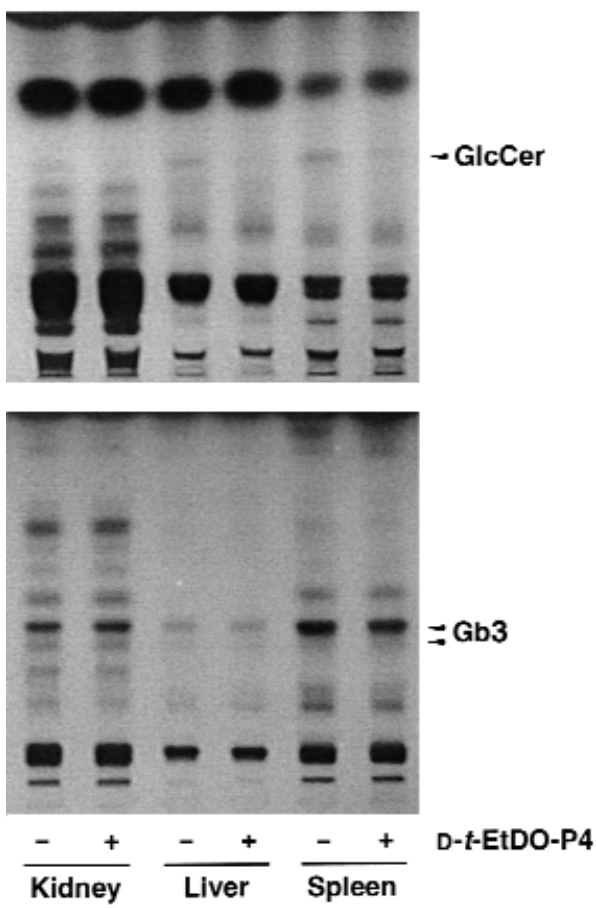

Figure 5

Effect of 3-day treatment with D-t-EtDO-P4 on glucosylceramide and Gb3 content in kidneys of $\alpha$-Gal $A^{-}$males. Glucosylceramide (GlcCer) and $\mathrm{Gb} 3$ were extracted and separated by the two-step solvent systems detailed in Methods. Glucosylceramide levels are visually decreased in the liver and spleens of the mice; no change in Gb3 levels was observed. 


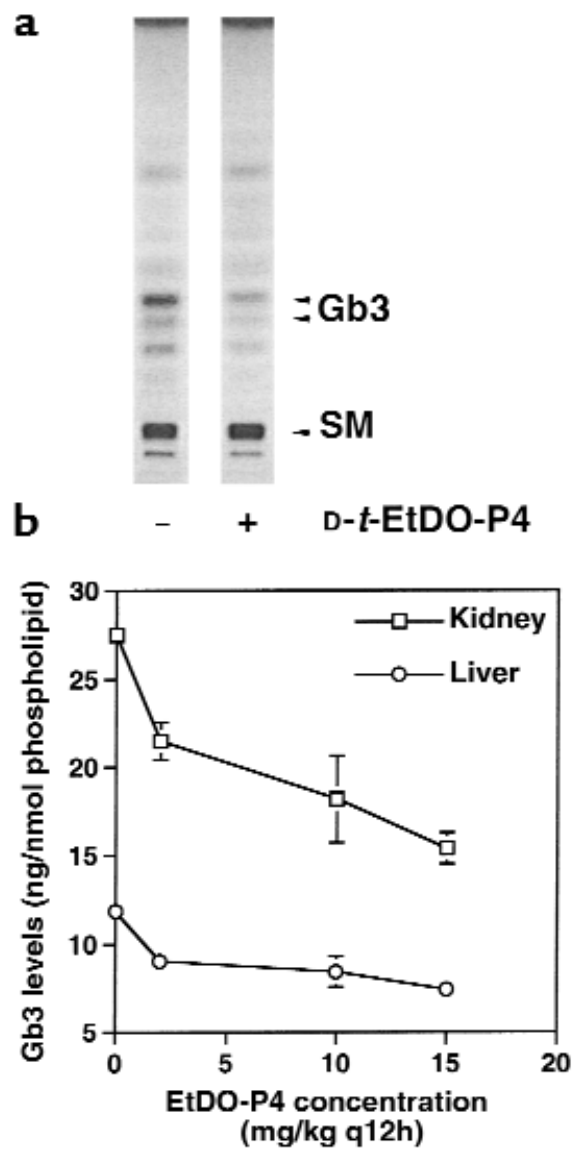

Figure 6

(a) Representative thin layer chromatogram of renal glycosphingolipids in $\alpha$-Gal $A^{-}$males treated for 4 weeks with $10 \mathrm{mg} / \mathrm{kg}$ of D- $t$-EtDO-P4 every 12 hours. (b) Concentration-dependent effects of D-t-EtDO-P4 on kidney and liver Gb3 content in $\alpha$-glactosidase-A-deficient mice treated for 4 weeks. SM, sphingomyelin.

fer in their ability to deplete glucosylceramide and elevate cell ceramide led to the development of more specific glucosylceramide synthase inhibitors. Phenyl substituted homologues, including D-t-EtDO-P4 and D-t-pOH-P4, potently inhibit glucosylceramide synthase at low nanomolar concentrations but only raise ceramide and inhibit cell growth at mid-micromolar concentrations (12). These findings suggest that there may exist a therapeutic window within which glucosylceramide- and glucosylceramide-based glycolipids could be lowered and ceramide-mediated toxicities could be avoided.

In the present study, the effects of a highly active glucosylceramide synthase inhibitor were studied in normal mice and in those with a targeted $\alpha$-galactosidase deficiency. The short-term treatment of mice with D-tEtDO-P4 resulted in a time- and concentrationdependent depletion of glucosylceramide in kidney, liver, and spleen. Glucosylceramide synthase inhibition was well tolerated, with no significant changes in body weight observed with doses as high as $15 \mathrm{mg} / \mathrm{kg}$ every 12 hours. The depletion of renal glucosylceramide was profound in both its extent and rapidity, consistent with a high basal rate of turnover for this glycolipid. A similar finding was reported in an earlier study with PDMP. PDMP was also observed to accumulate preferentially within the kidney (25). Thus, the marked renal effects may be the result of a similar pharmacokinetic profile for $\mathrm{D}-\mathrm{t}$-EtDO-P4. These findings suggest that the kidney in particular may be a good target for the use of this class of inhibitors.

By contrast, the effects on brain glucosylceramide were less profound. This finding may reflect the relatively poor distribution of D- $t$-EtDO-P4 into brain as was observed for PDMP (25). This may reflect an unfavorable pKa for both PDMP and D-t-EtDO-P4, which may limit the penetration of these drugs across the blood-brain barrier. This pharmacokinetic profile is preferable for Fabry disease or type I Gaucher disease in which the accumulation of glycolipids in the central nervous system is absent. However, the Fabry mouse is a poor model for determining the effects of glucosylceramide synthase inhibition on brain glycosphingolipids. More definitive data will need to be obtained in alternative models such as the Tay-Sachs and Sandhoff disease mice.

Two major toxicities associated with NBDNJ were
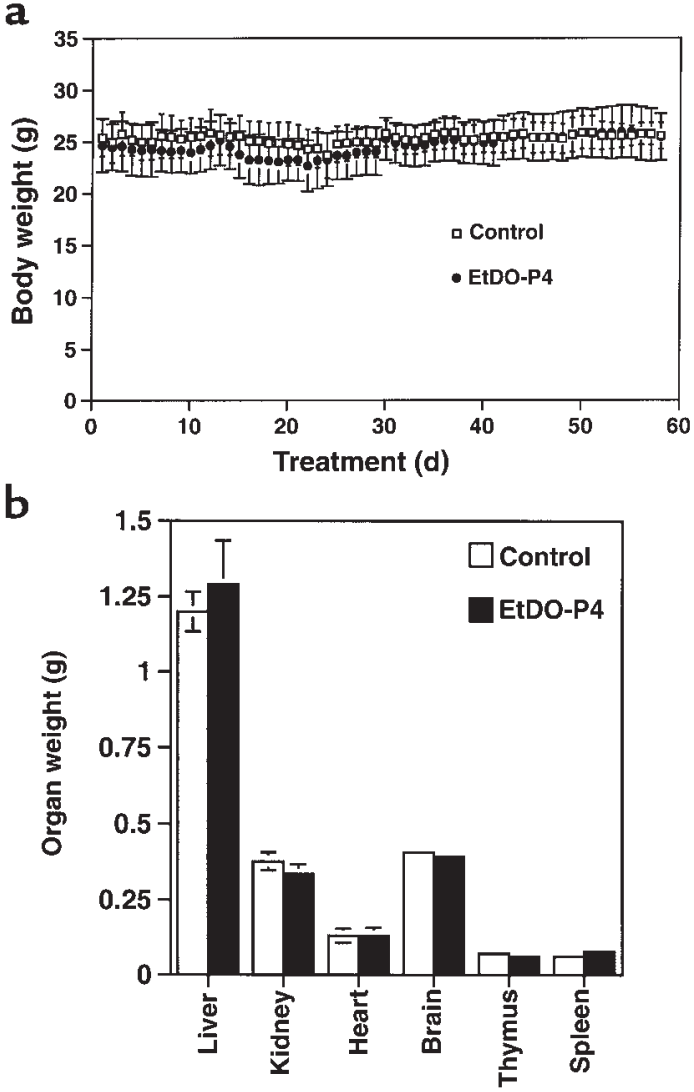

Figure 7

(a) Daily body weights of $\alpha$-Gal $A^{-}$males treated with $10 \mathrm{mg} / \mathrm{kg}$ of D-t-EtDO-P4 every 12 hours for 8 weeks. (b) Organ weights of $\alpha$-Gal $A^{-}$males treated with $10 \mathrm{mg} / \mathrm{kg}$ of D-t-EtDO-P4 every 12 hours for 8 weeks $(n=7)$. 


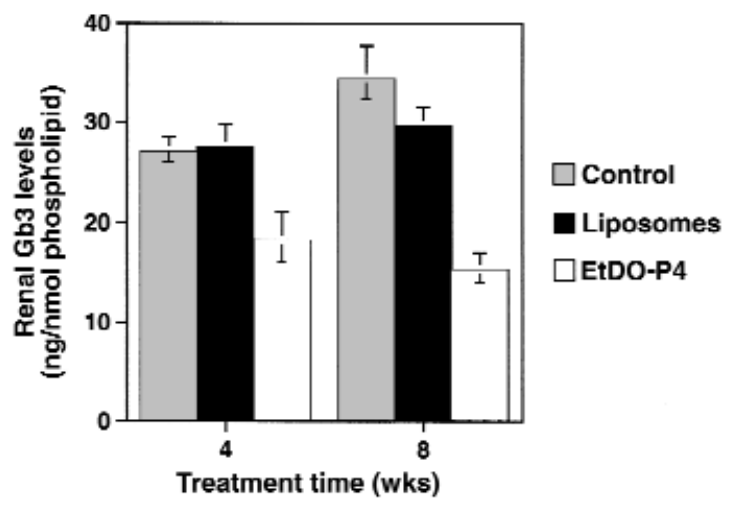

Figure 8

Gb3 content of kidney of $\alpha$-Gal $A^{-}$males treated for 4 or 8 weeks with $10 \mathrm{mg} / \mathrm{kg}$ of D-t-EtDO-P4 every 12 hours ( $n=5$ for 4-week treatments and $n=7$ for 8 -week treatments). $P<0.01$ by paired $t$ test for D-tEtDO-P4-treated versus liposome-treated mice.

not observed with D-t-EtDO-P4. Treated mice neither lost weight nor developed reversible acellularity of their lymphoid tissues. The former complication of NBDNJ was argued to be the result of malabsorption secondary to the inhibition of $\alpha$-glucosidase activity. Based on the current study, the lymphopenic response is arguably not the result of glucosylceramide synthase inhibition, as no change in spleen or thymus weights were observed even though significant depletion of glucosylceramide was measured in spleen.

The $\alpha$-Gal $A$ knockout mice provide a useful model of Fabry disease. These mice manifest a significant accumulation of Gb3 in their kidneys, hearts, and livers. In wild-type C57BL/6 mice, Gb3 is only detectable in kidney using thin layer chromatography and charring. Unfortunately, the knockout mice do not develop significant renal impairment within their first year of life, an end point of adult humans. Thus only tissue $\mathrm{Gb} 3$ content can be followed as a therapeutic end point in young mice.

When the $\alpha$-Gal A knockout mice were studied, short- term treatment with D-t-EtDO-P4 effectively lowered glucosylceramide, but little change in $\mathrm{Gb} 3$ was observed. However, after 4 weeks of treatment, renal Gb3 content was significantly lower than that of control animals. Importantly, D- $t$-EtDO-P4 not only prevented the further accumulation of renal Gb3 seen at 12- or 16-weekold mice, but lowered Gb3 to levels below that observed for 8-week-old mice.

Because no obvious decrement in more highly glycosylated glycosphingolipids were observed, this observation is consistent with several potential explanations. First, Gb3 may be lost from the kidneys as renal tubular epithelial cells are shed. Indeed, Gb3 content of renal epithelial cells recovered in the urine of humans with Fabry disease has been used as an measure of Gb3 burden (3). However, in the absence of direct tubular injury as may be seen with acute tubular necrosis, renal tubular epithelia does not turn over rapidly, as evidenced by a low number of mitotic figures observed in normal kidney. Alternatively, the exocytosis of lysosomes or of lysosomal contents may occur in a regulated fashion and may be enhanced in the D-t-EtDO-P4-treated mice. The localization of the lipid-rich vesicles to the apical aspects of the renal epithelia in the treated mice is consistent with this possibility. Another potential explanation for the reduction in Gb3 may be the existence of alternative degradative pathways for Gb3 that are $\alpha$-Gal A independent. Beutler and coworkers identified $\alpha$-Gal B as biochemically distinct from $\alpha$ Gal A (26). Dean and Sweeley demonstrated that $\alpha$ Gal B has glycosidase activity against Gb3, although it does not prevent Gb3 accumulation in Fabry disease (27). It is possible therefore that residual renal $\alpha$ Gal B activity lowers Gb3 when further synthesis is inhibited. Another potential pathway is a ceramide glycanase (28). This enzyme cleaves the entire glycan from glycosphingolipids but is inactive against cerebrosides. Mammalian ceramide glycanase activities have in fact been reported in the renal tissue of newborn rats (29). Whether such alternative pathways
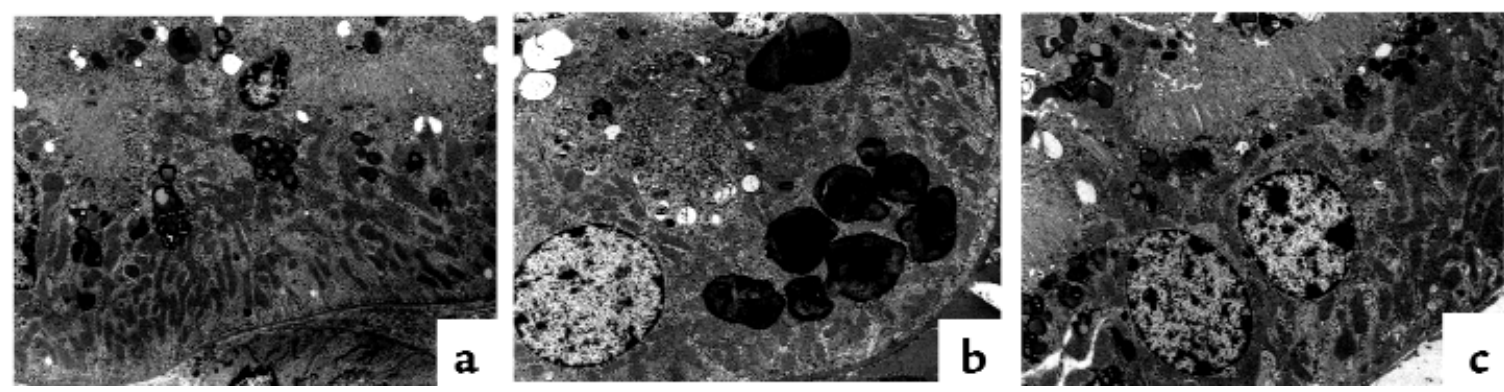

\section{Figure 9}

Electron micrographs of renal epithelial cells from $\alpha$-Gal $A^{-}$males treated with $10 \mathrm{mg} / \mathrm{kg}$ of liposomes or D-t-EtDO-P4 every 12 hours for 8 weeks. Representative electron micrographs of renal proximal tubular epithelial cells are demonstrated. (a) Proximal tubular cells from liposome-treated animals contained small myeloid bodies clustered in the apical cytoplasm and less frequently noted elsewhere in the cell. (b) Large lipid-laden inclusions were prominent, particularly toward the basal aspect of the cell. (c) Proximal tubular epithelial cells from D- $t$-EtDOP4-treated mice contained small lipid-rich inclusions in the apical cytoplasm immediately beneath the brush border and were virtually devoid of the large lamellated inclusion bodies. The original magnification of all photomicrographs is $\times 3,280$. 
may contribute to differences in clinical manifestations among humans with Fabry disease will need to be determined.

\section{Acknowledgments}

This work was supported by National Institutes of Health grants RO1 DK41487 and RO139255 and a Merit Review Research Award from the Veterans Administration (J.A. Shayman). The technical assistance of R. Kunkel in the performance of the ultrastructural studies is greatly appreciated.

1. Desnick, R.J., Ioannou, Y.A., and Eng, C.M. 1995. $\alpha$-Galactosidase A deficiency: Fabry disease. In The metabolic and molecular bases of inherited disease. 7th edition. C.R. Scriver, A.L. Beaudet, and W.S. Sly, editors. McGrawHill. New York, New York, USA. 2741-2784.

2. Beutler, E. 1997. Gaucher disease. Curr. Opin. Hematol. 4:19-23.

3. Schiffmann, R., et al. 2000. Infusion of alpha-galactosidase A reduces tissue globotriaosylceramide storage in patients with Fabry disease. Proc. Natl. Acad. Sci. USA. 97:365-370.

4. Radin, N.S. 1982. Inhibitors and stimulators of glucocerebroside metabolism. Prog. Clin. Biol. Res. 95:357-383.

5. Ratner, L., vander Heyden, N., and Dedera, D. 1991. Inhibition of HIV and SIV infectivity by blockade of alpha-glucosidase activity. Virology. 181:180-192.

6. Platt, F.M., et al. 1997. Prevention of lysosomal storage in Tay-Sachs mice treated with $N$-butyldeoxynojirimycin. Science. 276:428-431.

7. Jeyakumar, M., et al. 1999. Delayed symptom onset and increased life expectancy in Sandhoff disease mice treated with $N$-butyldeoxynojirimycin. Proc. Natl. Acad. Sci. USA. 96:6388-6393.

8. Platt, F.M., Reinkensmeier, G., Dwek, R.A., and Butters, T.D. 1997. Extensive glycosphingolipid depletion in the liver and lymphoid organs of mice treated with $N$-butyldeoxynojirimycin. J. Biol. Chem. 272:19365-19372.

9. Shayman, J.A., Lee, L., Abe, A., and Shu, L. 2000. Inhibitors of glucosylceramide synthase. Methods Enzymol. 311:373-387.

10. Abe, A., et al. 1992. Improved inhibitors of glucosylceramide synthase. J. Biochem. (Tokyo). 111:191-196.

11. Abe, A., et al. 1995. Structural and stereochemical studies of potent inhibitors of glucosylceramide synthase and tumor cell growth. J. Lipid Res. 36:611-621.

12. Lee, L., Abe, A., and Shayman, J.A. 1999. Improved inhibitors of glucosylceramide synthase. J. Biol. Chem. 274:14662-14669.
13. Abe, A., et al. 2000. Glycosphingolipid depletion in Fabry disease lymphoblasts with potent inhibitors of glucosylceramide synthase. Kidney Int. 57:446-454.

14. Ohshima, T., et al. 1997. alpha-Galactosidase A deficient mice: a model of Fabry disease. Proc. Natl. Acad. Sci. USA. 94:2540-2544.

15. Ames, B.N. 1966. Assay of inorganic phosphate, total phosphate, and phosphatases. Methods Enzymol. 8:115-118.

16. Liu, Y., et al. 1999. A genetic model of substrate deprivation therapy for a glycosphingolipid storage disorder. J. Clin. Invest. 103:497-505.

17. Tacconi, M.T., Lligona, L., Salmona, M., Pitsikas, N., and Algeri, S. 1991. Aging and food restriction: effect on lipids of cerebral cortex. Neurobiol. Aging. 12:55-59.

18. Vaswani, K.K., and Sharma, M. 1985. Effect of neonatal undernutrition on rat brain gangliosides. Int. J. Vitam. Nutr. Res. 55:323-329.

19. Vaswani, K.K. 1985. Effect of neonatal thiamine and vitamin A deficiency on rat brain gangliosides. Life Sci. 37:1107-1115.

20. Shukla, G.S., Shukla, A., Inokuchi, J., and Radin, N.S. 1991. Rapid kidney changes resulting from glycosphingolipid depletion by treatment with a glucosyltransferase inhibitor. Biochim. Biophys. Acta. 1083:101-108.

21. Rani, C.S., et al. 1995. Cell cycle arrest induced by an inhibitor of glucosylceramide synthase-correlation with cyclin-dependent kinases. J. Biol. Chem. 270:2859-2867.

22. Carson, K.G., Ganem, B., Radin, N.S., Abe, A., and Shayman, J.A. 1994 Studies on morpholinosphingolipids: potent inhibitors of glucosylceramide synthase. Tetrahedron Lett. 35:2659-2662.

23. Abe, A., Shayman, J.A., and Radin, N.S. 1996. A novel enzyme that catalyzes the esterification of $\mathrm{N}$-acetylsphingosine. J. Biol. Chem. 271:14383-14389.

24. Abe, A., and Shayman, J.A. 1998. Purification and characterization of 1 $O$-acylceramide synthase, a novel phospholipase A2. J. Biol. Chem. 273:8467-8474

25. Shukla, A., and Radin, N.S. 1991. Metabolism of D- $\left[{ }^{3} \mathrm{H}\right]$ threo-1-phenyl-2decanoylamino-3-morpholino-1-propanol, an inhibitor of glucosylceramide synthesis, and the synergistic action of an inhibitor of microsomal monooxygenase. J. Lipid Res. 32:713-722.

26. Beutler, E., Guinto, E., and Kuhl, W. 1973. Variability of $\alpha$-galactosidase $A$ and $B$ in different tissues of man. Am. J. Hum. Genet. 25:42-46.

27. Dean, K.J., and Sweeley, C.C. 1979. Studies on human liver $\alpha$-galactosidases. II. Purification and enzymatic properties of $\alpha$-galactosidase B. J. Biol. Chem. 254:10001-10005.

28. Li, S.C., DeGasperi, R., Muldrey, J.E., and Li, Y.T. 1986. A unique glycosphingolipid-splitting enzyme (ceramide-glycanase from Leech) cleaves the linkage between the oligosaccharide and the ceramide. Biochem. Biophys. Res. Commun. 141:346-352.

29. Basu, M., et al. 1997. Ceramide glycanase from rat mammary tissues: inhibition by PPMP(D-/L-) and its probable role in signal transduction. Indian J. Biochem. Biophys. 34:142-149. 